\title{
Comparison of testers in the selection of $\mathrm{S}_{3}$ families obtained from the UENF-14 variety of popcorn
}

\author{
Guilherme Ferreira Pena ${ }^{1}$, Antonio Teixeira do Amaral Júnior ${ }^{1 \star}$, Leandro Simões Azeredo Gonçalves², \\ Marcelo Vivas ${ }^{1}$, Rodrigo Moreira Ribeiro ${ }^{1}$, Gabrielle Sousa Mafra ${ }^{1}$, Adriano dos Santos ${ }^{1}$, \\ Carlos Alberto Scapim ${ }^{3}$ \\ 1. Universidade Estadual do Norte Fluminense Darcy Ribeiro - Centro de Ciências e Tecnologias Agropecuárias - Laboratório \\ de Melhoramento Genético Vegetal - Campos dos Goytacazes (RJ), Brazil. \\ 2. Universidade Estadual de Londrina - Departamento de Agronomia - Londrina (PR), Brazil. \\ 3. Universidade Estadual de Maringá - PGA Agronomia - Maringá (PR), Brazil.
}

\begin{abstract}
The use of topcross has proven to be an interesting option for the maize crop; however, for the popcorn, there is little information about the choice of the appropriate tester. In this context, this study aimed to analyze four testers including two open pollinated varieties(BRS Angela and UENF-14), one topcross hybrid (IAC125) and a line $\left(\mathrm{P}_{2}\right)$, to evaluate the combining ability of 50 S3 families of popcorn, obtained from the UENF-14 variety. Popcorn families were evaluated for grain yield (GY) and
\end{abstract}

popping expansion (PE). The estimates of general and specific combining abilities were obtained and the discrimination of each tester through differentiation index was carried out. The testers BRS Angela (for GY) and IAC 125 (for PE) were the most adequate, when combined with the best $\mathrm{S}_{3}$ families derived from UENF-14, for the production of popcorn hybrids for the Northern and Northwestern Fluminense Regions.

Key words: Zea mays var. everta, topcross, yield, popping expansion.

\section{INTRODUCTION}

Any breeding program focusing on successful hybrid combinations must concentrate its efforts to identify superior lines and their ability to transmit these desirable traits to the hybrids (Hallauer et al. 2010). In this context, diallel analysis is considered an important statistical tool for estimating parameters useful in selecting parents (Seifert et al. 2006; Barreto et al. 2012; Souza Neto et al. 2015).

However, a limiting factor of diallel analysis is the number of assessed parents, usually not exceeding ten, because of the effort to obtain the hybrids. In this case, the topcross technique, proposed by Davis (1927) and Jenkins and Brunson (1932), has proven to be a more appropriate option, aiming at overcoming the impossible assessment of progenitor lines in works with hybrids, involving a large number of lines.
The term "topcross" is used to denote crosses of lines with a tester. The tester can be a variety, a hybrid or even a line and receives this name for playing a role in evaluating the combining ability of the lines. Testers can be classified according to their genetic base (broad versus narrow), the degree of relatedness with the material evaluated (related versus unrelated) and their intrinsic genetic value (high pattern versus regular or inferior pattern) (Miranda Filho and Gorgulho 2001; Hallauer et al. 2010).

Despite the wide acceptance of the topcross method, the ideal tester selection process still remains a goal to be achieved by hybrid development programs, since issues of choice, type, number and efficiency of testers are perpetuated amidst theoretical and experimental studies (Ferreira et al. 2009). According to Hallauer et al. (2010), the choice of the tester should be on simplicity in use and generation of information that correctly classifies 
the relative potential of lines in cross, thus maximizing the genetic gain. According to Rodovalho et al. (2012), it is more important to use testers with broad genetic base (synthetic and open pollinated varieties) during the initial phase of breeding, since they test for general combining ability or additivity.

Compared to the common maize, there are few studies regarding the definition of testers for popcorn (Pinto et al. 2004; Viana et al. 2007; Scapim et al. 2008; Arnhold et al. 2009). An important detail that possibly hampers to choose the most suitable tester is the fact that the two main traits of the crop, popping expansion and grain yield, which should be evaluated together, have different mechanisms of inheritance (Pereira and Amaral Júnior 2001). Popping expansion is controlled by a small number of genes and there is predominance of additive gene action (Yongbin et al. 2012; Rodovalho et al. 2014). On the other hand, grain yield is genetically controlled by a large number of genes with predominance of non-additive gene effects.

Given these considerations, this study analyzed and compared four testers (BRS Angela, UENF-14, IAC 125 and $\mathrm{P}_{2}$ ) in the assessment of 50 partially inbred families $\left(\mathrm{S}_{3}\right)$, obtained from the UENF-14 variety of popcorn.

\section{MATERIAL AND METHODS}

For this study, $50 \mathrm{~S}_{3}$ families of popcorn were obtained from the variety UENF-14 of Universidade Estadual do Norte Fluminense Darcy Ribeiro (UENF) (Amaral Júnior et al. 2013). Four materials were evaluated as testers: BRS Angela (open pollinated variety), IAC 125 (topcross hybrid), $\mathrm{P}_{2}$ Line ( $\mathrm{S}_{7}$ generation coming from the CMS -42 composite of the Embrapa Maize and Sorghum) and UENF-14 (open pollinated variety). Hybrids were evaluated in the Experimental Station of PESAGRO-RIO, Itaocara, State of Rio de Janeiro, Northwestern Fluminense region ( $21^{\circ} 39^{\prime} \mathrm{S}$ latitude and $42^{\circ} 04^{\prime} \mathrm{W}$ longitude), in the growing season of 2013/2014.

Five trials were implemented in blocks with two replications, four for assessing the hybrids derived from crosses of families with each tester and the fifth for testing the performance of $S_{3}$ families. Each experimental unit consisted of a row of $3 \mathrm{~m}$, with spacing of $0.90 \mathrm{~m}$ between rows and five plants per meter. In the trials, the cultural practices, such as fertilization at planting and topdressing, irrigation, pest and weed control, among others, were made as required by the popcorn crop.

The traits evaluated were grain yield (GY) and popping expansion (PE). GY was assessed by measurement of the grain mass produced in each plot after discarding the cob and is expressed in $\mathrm{kg} \cdot \mathrm{ha}^{-1}$. In order to adapt the measurement of the yield, it was carried out the stand correction method, by analysis of covariance between the number of plants per plot and GY, according to the covariance of ideal stand methodology, proposed by Vencovsky and Barriga (1992). $\mathrm{PE}$ was determined by means of a seed sample, taken from the basal center of the ears (Granate et al. 2002) in each plot. All samples were sent to cold dry chamber to reach the equilibrium moisture content of 12 to $13 \%$. PE was determined in laboratory with the use of a microwave Panasonic NN-S65B, placing a sample of $30 \mathrm{~g}$ seed in a plastic container obtained in the USA, at the power of $1,000 \mathrm{~W}$ for 2 minutes and 30 seconds, in two replications per treatment. PE was expressed by the ratio between the volume of the popped popcorn, measured in a $2,000-\mathrm{mL}$ measuring cylinder, and the initial weight of grains ( $30 \mathrm{~g})$, being the final unit expressed in $\mathrm{mL} \cdot \mathrm{g}^{-1}$.

Statistical analysis of data followed the model of randomized blocks. The mean squares of treatment and the residue were used for the $\mathrm{F}$ test. The analysis of general and specific combining ability, based only on topcross crosses, was performed according to the scheme of analysis of variance in partial diallel at the level of average of treatments using the model proposed by Griffing (1956), adapted by Geraldi and Miranda Filho (1988). The efficiency of testers was evaluated by statistical indices of performance $(\mathrm{P})$ and differentiation (D) proposed by Fasoulas (1983). The P index gives the percentage, in relation to the number of means, that a particular cultivar statistically outperforms the others based on the minimum significant difference (MSD), determined by test of means. The $\mathrm{T}$ index gives the percentage of pairwise comparisons between cultivars that showed significant differences. Analyses were run using the software GENES (Cruz 2013).

\section{RESULTS AND DISCUSSION}

The mean squares of treatments (topcrosses) were significant at $5 \%$ probability for the four testers and 
families per se, indicating genetic variability for GY and $\mathrm{PE}$ (Table 1). The coefficients of variation $(\mathrm{CVe})$ for $\mathrm{GY}$ and $\mathrm{PE}$ were within acceptable limits for agricultural experimentation, except for the experiment assessing the lines per se that showed CVe values of 24.62 and 26.00, respectively, considered high (Scapim et al. 1995; Arnhold and Milani 2011; Fritsche Neto et al. 2012).

In the case of estimates of genetic parameters, the largest genetic variances were observed for the crossing BRS Angela $\times \mathrm{S}_{3}(22,830.61)$ and with the $\mathrm{S}_{3}$ families per se (0.93) for GY and PE, respectively (Table 1 ). The greatest increase in variability in topcross hybrids from the tester BRS Angela, for GY, can be explained because it is a broad base and unrelated tester, or because it presents many heterozygous loci. It is noteworthy that the high variability observed in $\mathrm{S}_{3}$ families per se, for $\mathrm{PE}$, is possibly related to the intrapopulation genetic variance, as they are individuals from the same population (UENF-14) (Amaral Júnior et al. 2013).

Considering the estimates of heritability, in general, their magnitudes remained very close independently of the tester. The exception applies to hybrids from the tester BRS Angela, specifically for PE, noting that this tester was not able to express the genetic variability among $\mathrm{S}_{3}$ families (Table 1).

There was a significant effect of general and specific combining abilities, estimated by diallel analysis of Griffing (1956), adapted by Geraldi and Miranda Filho (1988). The general and specific combining abilities of $\mathrm{S}_{3}$ families and testers have statistical significance, revealing differences for both traits between the values of the estimates of general $\left(\hat{G}_{i}\right)$ and specific $\left(\hat{S}_{i j}\right)$ combining abilities (Table 2). Regarding the general combining ability, the tester with the best estimate of $\hat{G}_{i}$ for GY was the $\mathrm{P}_{2}$

Table 1. Analysis of variance and estimates of genetic and phenotypic parameters for grain yield (in $\mathrm{kg} \cdot \mathrm{ha}^{-1}$ ) and popping expansion (PE, in $\mathrm{mL} \cdot \mathrm{g}^{-1}$ ) of topcross hybrids and $\mathrm{S}_{3}$ families per se.

\begin{tabular}{|c|c|c|c|c|c|c|}
\hline \multirow[b]{2}{*}{ SV } & \multirow[b]{2}{*}{ DF } & \multicolumn{5}{|c|}{ Mean squares } \\
\hline & & BRS Angela & IAC 125 & $\mathbf{P}_{2}$ & UENF14 & $\mathbf{S}_{3}$ per se \\
\hline & & \multicolumn{5}{|c|}{ Grain yield } \\
\hline Repetitions & 1 & 2183900.99 & 593525.18 & 343761.99 & 370365.60 & 76517.79 \\
\hline Treatments & 49 & $1219809.45^{\star \star}$ & $1178969.05^{\star \star}$ & $1056164.30^{*}$ & $1127949.90^{\star \star}$ & $398531.47^{\star \star}$ \\
\hline Residual & 49 & 489229.97 & 573285.61 & 540400.29 & 544026.16 & 122655.83 \\
\hline Mean & & 4638.79 & 4531.62 & 5264.58 & 4353.59 & 1346.69 \\
\hline$\hat{\sigma}_{G}^{2}$ & & 22830.61 & 18927.61 & 16117.62 & 18247.62 & 8621.11 \\
\hline$\sigma_{F}^{2}$ & & 38119.04 & 36842.78 & 33005.13 & 35248.43 & 12454.11 \\
\hline$h^{2}$ & & 0.59 & 0.51 & 0.49 & 0.51 & 0.69 \\
\hline $\mathrm{CV}_{\mathrm{e}}$ & & 15.07 & 16.71 & 13.96 & 16.94 & 26.00 \\
\hline $\mathrm{CV}_{\mathrm{g}}$ & & 3.25 & 3.03 & 2.41 & 3.10 & 6.89 \\
\hline \multirow[t]{2}{*}{$\mathrm{I}_{\mathrm{v}}(\%)$} & & 21.61 & 18.17 & 17.27 & 18.31 & 26.52 \\
\hline & & \multicolumn{5}{|c|}{ Popping expansion } \\
\hline Repetitions & 1 & 0.24 & 1.62 & 14.31 & 3.05 & 32.12 \\
\hline Treatments & 49 & $23.21^{\star *}$ & $25.04^{\star \star}$ & $27.60^{\star *}$ & $18.35^{\star \star}$ & $67.87^{\star}$ \\
\hline Residual & 49 & 19.46 & 10.89 & 13.58 & 7.85 & 38.11 \\
\hline Mean & & 30.99 & 32.13 & 25.44 & 30.13 & 25.06 \\
\hline$\sigma_{G}^{2}$ & & 0.11 & 0.44 & 0.44 & 0.33 & 0.93 \\
\hline$\sigma_{F}^{2}$ & & 0.72 & 0.78 & 0.86 & 0.57 & 2.12 \\
\hline$h^{2}$ & & 0.16 & 0.56 & 0.51 & 0.57 & 0.44 \\
\hline $\mathrm{CV}_{\mathrm{e}}$ & & 14.23 & 14.22 & 14.48 & 9.3 & 24.62 \\
\hline $\mathrm{CV}_{\mathrm{g}}$ & & 1.10 & 2.07 & 2.60 & 1.90 & 3.85 \\
\hline $\mathrm{I}_{\mathrm{v}}(\%)$ & & 7.76 & 14.55 & 17.97 & 20.44 & 15.63 \\
\hline
\end{tabular}

*,**Significant at 5 and $1 \%$ probability, respectively; SV = Sources of variation; DF = Degrees of freedom; $\hat{\sigma}_{\mathrm{G}}^{2}=$ Genotypic variance; $\hat{\sigma}_{\mathrm{F}}^{2}=$ Fenotypic variance; $\hat{h}^{2}=$ Heritability; $\mathrm{CV}_{\mathrm{e}}=$ Experimental variance coefficient; $\mathrm{CV}_{\mathrm{g}}=$ Genetic variance coefficient; $\mathrm{I}_{\mathrm{v}}(\%)=$ Variation index 
Line, while, for the PE, testers IAC 125 and BRS Angela achieved the best estimates, reflecting the greater allelic complementarity of these testers with $\mathrm{S}_{3}$ families (Figure 1). Barreto et al. (2012) evaluated the combining ability of $\mathrm{S}_{2}$ families of popcorn and also obtained a similar result for the tester IAC 125, indicating it as the tester with the best general combining ability (GCA) indices for PE.

$\mathrm{S}_{3}$ families with higher estimates of GCA were 9, 33, 4, 20 and 16 for GY, and 18, 23, 42, 11, and 25 for PE (Figure 2). Among the $50 \mathrm{~S}_{3}$ families, only 9,16 and 23 stood out for concomitantly presenting significantly positive estimates of GCA for GY and PE and should be evaluated more carefully in the breeding program conducted in the Northern and Northwestern Fluminense Regions by UENF.

As for the specific combining ability of $\mathrm{S}_{3}$ families, for crosses with the tester BRS Angela, families 21, 4, 13, 50, 22 and 45 demonstrated the highest values of specific combining ability (SCA); for the tester IAC 125, the families 9, 20, 11, 35, 3 and 45 stood out. For tester $\mathrm{P}_{2}$, families 49, 43, 24, 41, 18 and 50 showed the highest SCA indices and, in relation to the tester UENF-14, families 19, 2, 4, 45, 48 and 47 were those with higher estimates of $\hat{S}_{i j}$ (Table 3 ). The two hybrids showing better estimates of the effects of SCA were derived from crosses between tester $\mathrm{P}_{2}$ and family 49 and between the tester IAC 125 and family 9 . These hybrids, respectively, had $\hat{S}_{i j}$ effects of 2,359 and 1,960, besides having values around $7,400 \mathrm{~kg} \cdot \mathrm{ha}^{-1}$. These two materials are very interesting because they express high genetic non-additive effects in their genotypes, derived from allelic complementation of their parents, and will certainly comprise hybrids with high average GY, which may be useful in interpopulation breeding programs in popcorn.

According to the SCA estimates for PE, families with better performance in relation to the tester BRS Angela were the genotypes 19,44, 39, 30, 31 and 50. As for the

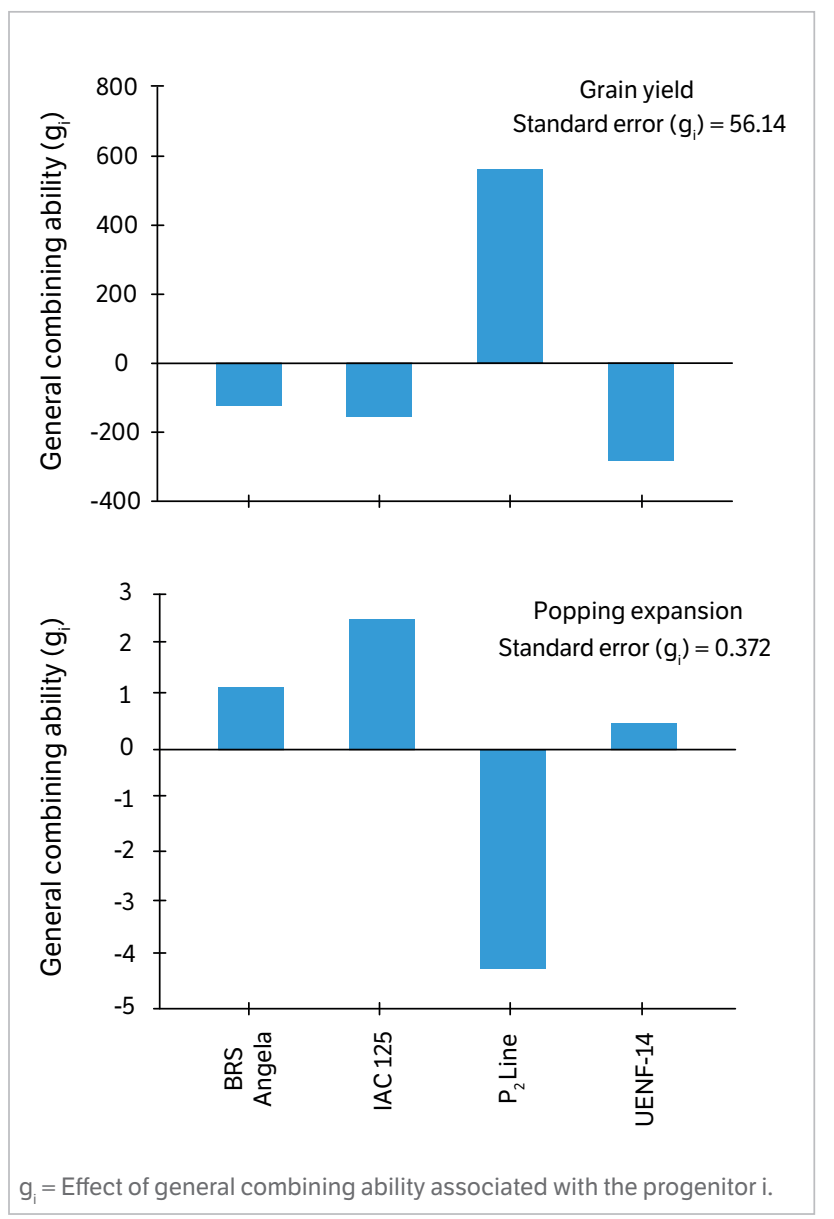

Figure 1. Estimates of the effects of general combining ability $\left(\hat{G}_{i}\right)$ associated with testers for grain yield $\left(\mathrm{kg} \cdot \mathrm{ha}^{-1}\right)$ and popping expansion ( $\mathrm{mL} \cdot \mathrm{g}^{-1}$ ) according to Griffing (1956).

Table 2. Partial diallel analysis of means of treatments for grain yield and popping expansion in the study of general and specific combining abilities.

\begin{tabular}{cccc} 
SV & DF & GY & MS \\
\cline { 3 - 4 } & & $1635761.22^{\star \star}$ & $35.25^{\star \star}$ \\
\hline Crossings & 199 & $1938796.04^{\star \star}$ & $25.25^{\star \star}$ \\
\hline GCA S Families (I) & 49 & $19506622.89^{\star \star}$ & $883.32^{\star \star}$ \\
\hline GCA Testers (II) & 3 & $1170038.15^{\star \star}$ & $21.28^{\star \star}$ \\
\hline SCA & 147 & 536735.51 & 12.94 \\
\hline Residual & 196 & & \\
\hline
\end{tabular}

**Significant $1 \%$ probability; MS = Mean squares; $S V=$ Sources of variation; $D F=$ Degrees of freedom; $G Y=$ Grain yield; $P E=$ Popping expansion; $G C A=G e n e r a l$ combining ability; SCA = Specific combining ability. 
tester IAC 125, families with higher indices were 2, 49, 17, 46, 44 and 39. For $\mathrm{P}_{2}$ tester, families with greater SCA effects were 2, 30, 1, 39, 45 and 46 and, for the tester UENF-14, families 26, 22, 1, 20, 40 and 37 stood out with the highest indices of $\hat{S}_{i j}$ (Table 4). Among the better crosses in relation to SCA, with high GCA values and relevant estimates of PE, for the tester BRS Angela, it can be highlighted family 18 with PE of $35 \mathrm{~mL} \cdot \mathrm{g}^{-1}$; for the tester IAC 125 , family 23 , with PE of $36.67 \mathrm{~mL} \cdot \mathrm{g}^{-1}$; and, for the tester UENF-14, family 11 , with $33 \mathrm{~mL} \cdot \mathrm{g}^{-1}$ (Table 4). By different mechanisms of genetic action of PE (additive and non-additive) which determine respectively the GCA and SCA, it can be inferred that these crossings are interesting and also deserve attention from the popcorn breeding program of UENF.

Higher values for differentiation index (D) of Fasoulas (1983), for GY, were obtained by tester UENF-14
( $\mathrm{D}=28.98)$ and BRS Angela $(\mathrm{D}=28.24)$, indicating these testers as having greater efficiency in discriminating $\mathrm{S}_{3}$ families (Table 3). Rodovalho et al. (2012) examined different testers in the discrimination of $64 \mathrm{~S}_{2}$ families of popcorn, for GY, and also indicated the tester BRS Angela using this differentiation index. Considering the trait PE, the highest differentiation indices, 25.22 and $19.92 \%$, were obtained, respectively, for the testers UENF-14 and IAC 125, indicating that these parents discriminated better the trait $\mathrm{PE}$ of $\mathrm{S}_{3}$ families.

Among the testers used, the $\mathrm{P}_{2}$ Line exhibited only one hybrid of high GY by combining with one of the seven $\mathrm{S}_{3}$ families of greater values of GCA, the family 2 . The BRS Angela was the tester that concomitantly classified most high-yield hybrids with $\mathrm{S}_{3}$ families of high GCA. Of the seven $\mathrm{S}_{3}$ families with the highest GCA indices, five that comprised the topcross hybrids of higher yields,

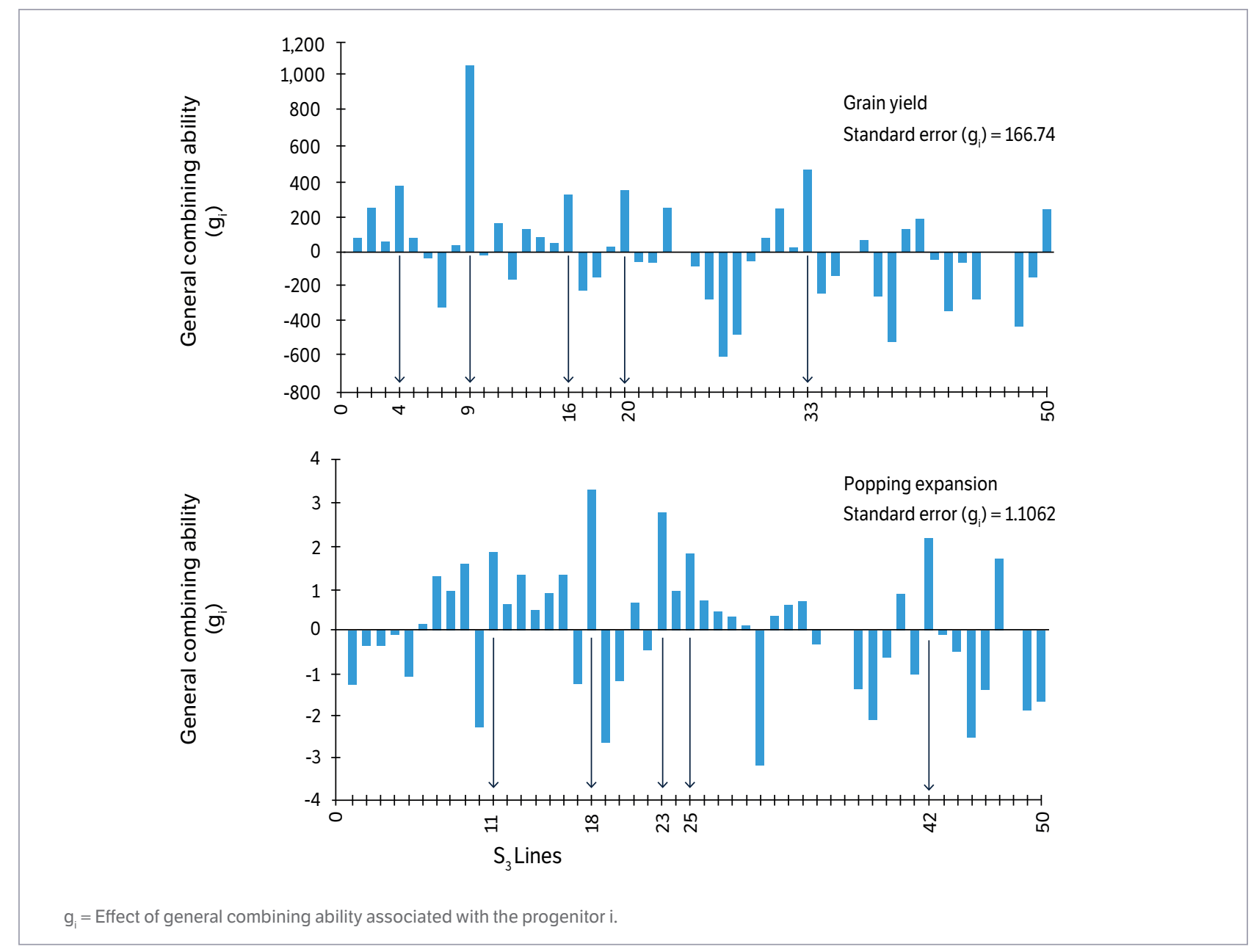

Figure 2. Estimates of the effects of general combining ability $\left(\hat{G}_{i}\right)$ associated with $\mathrm{S}_{3}$ families for grain yield $\left(\mathrm{kg} \cdot \mathrm{ha} \mathrm{a}^{-1}\right)$ and popping expansion $\left(\mathrm{mL} \cdot \mathrm{g}^{-1}\right)$ according to Griffing (1956). 
Table 3. Means, estimates of specific combining ability $\left(\hat{S}_{\mathrm{i}}\right)$ and discrimination ability of testers, according to the $D$ index and P performance index (Fasoulas 1983), for grain yield $\left(\mathrm{kg}^{\prime \prime} \cdot \mathrm{ha}^{-1}\right)$ of topcross hybrids, based on the Student's t-test (0.05) for means comparisons.

\begin{tabular}{|c|c|c|c|c|c|c|c|c|c|c|c|c|}
\hline \multirow{2}{*}{ Order } & \multicolumn{3}{|c|}{ BRS Angela } & \multicolumn{3}{|c|}{ IAC 125} & \multicolumn{3}{|c|}{$\mathbf{P}_{2}$} & \multicolumn{3}{|c|}{ UENF-14 } \\
\hline & $\mathbf{S}_{3}$ & Mean & $\hat{\boldsymbol{S}}_{i j}$ & $\mathrm{~S}_{3}$ & Mean & $\hat{\boldsymbol{S}}_{i j}$ & $\mathbf{S}_{3}$ & Mean & $\hat{\boldsymbol{S}}_{i j}$ & $\mathbf{S}_{3}$ & Mean & $\hat{\boldsymbol{S}}_{i j}$ \\
\hline $1^{\circ}$ & 4 & 5113.81 & 1463.8 & 9 & 7444.44 & 1960.3 & 49 & 7349.70 & 2359 & 2 & 6803.70 & 1223.7 \\
\hline $2^{\circ}$ & 9 & 5112.79 & 771.9 & 20 & 6481.48 & 1712.5 & 41 & 6768.82 & 1445 & 19 & 6039.06 & 1719.6 \\
\hline $3^{\circ}$ & 13 & 4790.06 & 1387.6 & 11 & 5577.78 & 996.7 & 24 & 6589.84 & 1460 & 4 & 5837.04 & 1169.4 \\
\hline $4^{\circ}$ & 50 & 4751.87 & 1235.2 & 33 & 5574.07 & 689.1 & 43 & 6580.71 & 1785 & 20 & 5352.94 & 709.6 \\
\hline $5^{\circ}$ & 16 & 4583.67 & 981.8 & 3 & 5451.85 & 976 & 50 & 6454.00 & 1074 & 47 & 5296.88 & 957 \\
\hline $6^{\circ}$ & 22 & 4359.79 & 1149.8 & 14 & 5374.07 & 875.5 & 18 & 6370.02 & 1375 & 46 & 5251.93 & -1032 \\
\hline $7^{\circ}$ & 15 & 4195.29 & 871.0 & 31 & 5346.30 & 681 & 2 & 6235.66 & 846.5 & 16 & 5238.64 & 619.1 \\
\hline $8^{\circ}$ & 23 & 4160.94 & 634.1 & 35 & 5274.07 & 993.5 & 30 & 6120.85 & 903 & 44 & 5238.64 & -563.6 \\
\hline $9^{\circ}$ & 33 & 4150.85 & 409.2 & 37 & 5222.22 & 736.8 & 36 & 6102.33 & 965.5 & 23 & 5174.58 & 630.1 \\
\hline $10^{\circ}$ & 30 & 4128.63 & 774.0 & 45 & 5088.89 & 943.0 & 13 & 6012.06 & 746.3 & 21 & 5076.77 & 842.2 \\
\hline $11^{\circ}$ & 31 & 4111.77 & 589.7 & 25 & 5081.48 & 747.9 & 1 & 5846.00 & 628.6 & 30 & 5072.98 & 700.7 \\
\hline $12^{\circ}$ & 45 & 4108.45 & 1106 & 5 & 5000.00 & 504.6 & 10 & 5830.41 & 715.2 & 24 & 4976.69 & 294.2 \\
\hline $13^{\circ}$ & 41 & 4002.19 & -459.7 & 23 & 4970.37 & 300.3 & 15 & 5823.77 & 636.2 & 3 & 4954.97 & 604.7 \\
\hline $14^{\circ}$ & 7 & 3964.13 & 1007.4 & 41 & 4855.56 & 251.9 & 31 & 5783.81 & 398.5 & 9 & 4932.24 & -426.2 \\
\hline $15^{\circ}$ & 6 & 3930.67 & 695.7 & 4 & 4851.85 & 58.6 & 29 & 5734.88 & 653.4 & 31 & 4883.51 & -1104 \\
\hline $16^{\circ}$ & 17 & 3874.61 & 819.4 & 42 & 4840.74 & 470.9 & 11 & 5719.29 & 418.2 & 15 & 4875.67 & 533.7 \\
\hline $17^{\circ}$ & 8 & 3811.64 & 499.3 & 1 & 4829.63 & 332.2 & 33 & 5608.96 & 4.0 & 8 & 4832.24 & 502.2 \\
\hline $18^{\circ}$ & 3 & 3807.43 & -603.9 & 18 & 4829.63 & 555.3 & 17 & 5583.98 & 665.5 & 32 & 4775.59 & 459.2 \\
\hline $19^{\circ}$ & 19 & 3775.24 & -604.8 & 19 & 4662.96 & 217.8 & 8 & 5537.81 & 362.2 & 10 & 4725.85 & 456.2 \\
\hline $20^{\circ}$ & 40 & 3749.51 & 344.7 & 50 & 4655.56 & -4.4 & 9 & 5511.11 & -5.7 & 11 & 4712.71 & 257.3 \\
\hline $21^{\circ}$ & 2 & 3722.37 & 196.5 & 46 & 4625.93 & 205.2 & 44 & 5504.05 & 430.7 & 6 & 4648.65 & 396 \\
\hline $22^{\circ}$ & 46 & 3637.06 & 359.6 & 8 & 4618.52 & 162.9 & 40 & 5500.78 & 232.7 & 33 & 4628.03 & -131.2 \\
\hline $23^{\circ}$ & 1 & 3627.61 & 273.5 & 6 & 4607.41 & 229.1 & 14 & 5464.69 & 246.1 & 34 & 4591.50 & 539 \\
\hline $24^{\circ}$ & 20 & 3549.32 & -76.3 & 16 & 4511.11 & -234 & 35 & 5418.52 & 418 & 1 & 4493.86 & 122.1 \\
\hline $25^{\circ}$ & 14 & 3539.23 & 184.0 & 36 & 4496.30 & 79.4 & 23 & 5377.78 & -12.2 & 50 & 4453.96 & 38.7 \\
\hline $26^{\circ}$ & 32 & 3515.47 & 216.7 & 30 & 4492.59 & -5.3 & 4 & 5352.80 & -160 & 22 & 4409.94 & 182.3 \\
\hline $27^{\circ}$ & 21 & 3488.01 & 1517.1 & 15 & 4488.89 & 21.3 & 19 & 5331.96 & 166.8 & 13 & 4373.49 & -46.5 \\
\hline $28^{\circ}$ & 37 & 3406.41 & 64.3 & 32 & 4474.07 & 32.06 & 42 & 5317.92 & 228.1 & 37 & 4368.27 & 8.5 \\
\hline $29^{\circ}$ & 5 & 3378.44 & 26.3 & 47 & 4429.63 & 3.06 & 25 & 5208.96 & 155.4 & 40 & 4362.96 & 317 \\
\hline $30^{\circ}$ & 42 & 3369.24 & 142.7 & 2 & 4400.00 & -269.1 & 5 & 5207.41 & -7.9 & 49 & 4183.59 & -415 \\
\hline $31^{\circ}$ & 36 & 3356.21 & 82.6 & 49 & 4385.19 & 114.6 & 34 & 5168.99 & 271 & 29 & 4118.52 & -117.3 \\
\hline $32^{\circ}$ & 11 & 3329.14 & -1094 & 26 & 4377.78 & 232.4 & 47 & 5061.01 & -85.5 & 28 & 4107.41 & 286.3 \\
\hline $33^{\circ}$ & 38 & 3270.52 & 254.2 & 44 & 4225.93 & -127.3 & 3 & 4991.22 & -204 & 39 & 4093.10 & 6 \\
\hline $34^{\circ}$ & 34 & 3260.94 & -590.7 & 39 & 4137.04 & 235.3 & 38 & 4977.18 & 97.6 & 26 & 4058.67 & 38.9 \\
\hline $35^{\circ}$ & 43 & 3252.00 & -1023 & 12 & 4051.85 & -207.3 & 16 & 4963.14 & -502 & 12 & 3942.34 & -191.2 \\
\hline $36^{\circ}$ & 35 & 3251.36 & 114.2 & 24 & 4033.33 & -376.8 & 37 & 4807.58 & -398 & 7 & 3805.22 & -169.1 \\
\hline $37^{\circ}$ & 39 & 3181.63 & -740.6 & 10 & 4000.00 & -395.2 & 28 & 4754.18 & 87.5 & 14 & 3799.92 & -573 \\
\hline $38^{\circ}$ & 10 & 3164.13 & -87.8 & 34 & 3970.37 & -207.7 & 20 & 4719.89 & -769 & 25 & 3760.86 & -447.0 \\
\hline $39^{\circ}$ & 26 & 2980.99 & -21.06 & 48 & 3951.85 & -36.9 & 12 & 4664.51 & -314 & 41 & 3747.06 & -59.4 \\
\hline $40^{\circ}$ & 18 & 2849.70 & -281.3 & 38 & 3940.74 & -218.8 & 32 & 4583.03 & -580 & 36 & 3730.64 & -560.5 \\
\hline $41^{\circ}$ & 25 & 2846.64 & -343.5 & 13 & 3770.37 & -775.3 & 45 & 4562.19 & -303 & 18 & 3495.20 & -653.4 \\
\hline $42^{\circ}$ & 49 & 2842.42 & -1315 & 40 & 3729.63 & -818.4 & 6 & 4436.44 & -662 & 48 & 3448.15 & 996 \\
\hline $43^{\circ}$ & 47 & 2780.35 & -502.9 & 29 & 3585.19 & -776.2 & 7 & 4434.88 & -385 & 38 & 3423.23 & -610 \\
\hline $44^{\circ}$ & 27 & 2630.67 & -42.8 & 43 & 3540.74 & -534.2 & 27 & 4426.53 & -110 & 5 & 3416.92 & -952.8 \\
\hline $45^{\circ}$ & 48 & 2622.24 & -223.2 & 22 & 3496.30 & -856.9 & 48 & 4401.55 & -307 & 35 & 3407.91 & -747 \\
\hline $46^{\circ}$ & 12 & 2529.27 & -586.6 & 21 & 3340.74 & -1019 & 26 & 4284.58 & -581 & 43 & 3385.69 & -1084 \\
\hline $47^{\circ}$ & 24 & 2231.31 & -1035 & 28 & 3255.56 & -691.1 & 46 & 4283.21 & -857 & 27 & 3306.82 & -384.3 \\
\hline $48^{\circ}$ & 28 & 2121.22 & -682.2 & 27 & 3122.22 & -694.6 & 21 & 4095.09 & -985 & 42 & 3159.77 & -731 \\
\hline $49^{\circ}$ & 44 & 2113.30 & -1096 & 7 & 2966.67 & -233.3 & 22 & 3883.98 & -120 & 17 & 3150.17 & -922.7 \\
\hline \multirow[t]{2}{*}{$50^{\circ}$} & 29 & 1125.56 & -2092 & 17 & 2651.85 & -880 & 39 & 3829.80 & -792 & 45 & 2988.30 & 1011 \\
\hline & \multicolumn{3}{|c|}{$D=28,24$} & \multicolumn{3}{|c|}{$D=26.53$} & \multicolumn{3}{|c|}{$D=27.18$} & \multicolumn{3}{|c|}{$\mathrm{D}=28.98$} \\
\hline
\end{tabular}


Table 4. Means, estimates of specific combining ability $\left(\hat{S}_{i j}\right)$ and discrimination ability of testers, according to the $D$ index and $P$ performance index (Fasoulas 1983), for popping expansion $\left(\mathrm{mL} \cdot \mathrm{g}^{-1}\right)$ of topcross hybrids, based on the Student's t-test (0.05) for means comparisons.

\begin{tabular}{|c|c|c|c|c|c|c|c|c|c|c|c|c|}
\hline \multirow{2}{*}{ Order } & \multicolumn{3}{|c|}{ BRS Angela } & \multicolumn{3}{|c|}{ IAC 125} & \multicolumn{3}{|c|}{$\mathbf{P}_{2}$} & \multicolumn{3}{|c|}{ UENF-14 } \\
\hline & $\mathrm{S}_{3}$ & Mean & $\hat{\boldsymbol{S}}_{i j}$ & $\mathrm{~S}_{3}$ & Mean & $\hat{\boldsymbol{S}}_{i j}$ & $\mathrm{~S}_{3}$ & Mean & $\hat{S}_{i j}$ & $\mathrm{~S}_{3}$ & Mean & $\hat{\boldsymbol{S}}_{i j}$ \\
\hline $1^{\circ}$ & 44 & 37.33 & 7.08 & 21 & 41.33 & -4 & 2 & 34.17 & 9.22 & 40 & 34.83 & 3.93 \\
\hline $2^{\circ}$ & 39 & 36.67 & 6.56 & 2 & 40.67 & 8.96 & 1 & 31.92 & 7.88 & 25 & 34.58 & 2.74 \\
\hline $3^{\circ}$ & 7 & 36.25 & 4.2 & 45 & 40.00 & 3.04 & 7 & 30.58 & 4.01 & 4 & 33.83 & 3.88 \\
\hline $4^{\circ}$ & 31 & 35.83 & 4.76 & 23 & 36.67 & 1.83 & 30 & 30.50 & 8.37 & 47 & 33.58 & 1.85 \\
\hline $5^{\circ}$ & 19 & 35.42 & 7.3 & 48 & 36.25 & 0.39 & 39 & 30.42 & 5.75 & 1 & 33.33 & 4.55 \\
\hline $6^{\circ}$ & 18 & 35.00 & 0.95 & 44 & 35.42 & 3.84 & 18 & 29.33 & 0.73 & 11 & 33.00 & 1.11 \\
\hline $7^{\circ}$ & 48 & 34.67 & 3.88 & 9 & 35.00 & 1.37 & 8 & 29.25 & 3.03 & 20 & 32.92 & 4.05 \\
\hline $8^{\circ}$ & 13 & 34.58 & 2.54 & 39 & 35.00 & 3.57 & 46 & 29.00 & 5.09 & 27 & 32.83 & 2.35 \\
\hline $9^{\circ}$ & 16 & 34.25 & 2.19 & 25 & 34.58 & 0.72 & 45 & 28.25 & 5.47 & 14 & 32.58 & 2.07 \\
\hline $10^{\circ}$ & 34 & 34.00 & 2.49 & 14 & 34.58 & 2.05 & 23 & 28.08 & 0.01 & 37 & 32.58 & 3.91 \\
\hline $11^{\circ}$ & 33 & 33.92 & 3.6 & 46 & 34.58 & 3.92 & 12 & 27.75 & 1.83 & 15 & 32.42 & 1.49 \\
\hline $12^{\circ}$ & 2 & 33.83 & 3.4 & 33 & 34.50 & 1.76 & 27 & 27.58 & 1.84 & 22 & 32.33 & 5.41 \\
\hline $13^{\circ}$ & 21 & 33.75 & 2.3 & 35 & 34.50 & 2.39 & 49 & 27.58 & 4.15 & 23 & 32.33 & -0.5 \\
\hline $14^{\circ}$ & 26 & 33.67 & 2.22 & 49 & 34.50 & 4.31 & 9 & 27.42 & 0.55 & 31 & 32.33 & 1.95 \\
\hline $15^{\circ}$ & 14 & 33.42 & 2.2 & 41 & 34.33 & 3.30 & 16 & 27.33 & -6.8 & 8 & 32.25 & 1.29 \\
\hline $16^{\circ}$ & 23 & 33.33 & -0.2 & 47 & 34.17 & 0.42 & 40 & 27.25 & 1.09 & 34 & 32.00 & 2.3 \\
\hline $17^{\circ}$ & 50 & 33.33 & 4.25 & 37 & 33.42 & 2.73 & 21 & 27.17 & 1.23 & 48 & 31.92 & 1.83 \\
\hline $18^{\circ}$ & 30 & 33.08 & 5.52 & 6 & 33.33 & 1.12 & 48 & 27.00 & 1.66 & 45 & 31.42 & 3.89 \\
\hline $19^{\circ}$ & 47 & 32.92 & 0.48 & 24 & 33.33 & 0.34 & 33 & 26.92 & 0.94 & 50 & 31.33 & 2.95 \\
\hline $20^{\circ}$ & 5 & 32.67 & 3.01 & 34 & 33.33 & 1.60 & 5 & 26.50 & 2.28 & 49 & 31.17 & 3 \\
\hline $21^{\circ}$ & 9 & 32.42 & 0.11 & 40 & 33.33 & 0.41 & 28 & 26.50 & 0.88 & 16 & 31.00 & -0.3 \\
\hline $22^{\circ}$ & 43 & 32.33 & -5.2 & 42 & 33.17 & -1.1 & 36 & 26.25 & 0.96 & 39 & 30.92 & 1.50 \\
\hline $23^{\circ}$ & 46 & 32.08 & 2.73 & 4 & 33.17 & 1.19 & 4 & 26.08 & 0.87 & 26 & 30.75 & 5.92 \\
\hline $24^{\circ}$ & 35 & 32.00 & -8.4 & 8 & 33.00 & 0.02 & 14 & 25.92 & 0.15 & 35 & 30.67 & 0.58 \\
\hline $25^{\circ}$ & 45 & 32.00 & -3.9 & 15 & 32.92 & -0.1 & 42 & 25.58 & -1.9 & 44 & 30.33 & 0.77 \\
\hline $26^{\circ}$ & 11 & 31.92 & -0.6 & 20 & 32.83 & 1.95 & 15 & 25.42 & -0.7 & 7 & 30.00 & -1.3 \\
\hline $27^{\circ}$ & 32 & 31.67 & 0.32 & 31 & 32.83 & 0.43 & 22 & 25.08 & 0.24 & 24 & 29.83 & -1.1 \\
\hline $28^{\circ}$ & 25 & 30.92 & -1.6 & 36 & 32.67 & -9.1 & 13 & 24.92 & -1.7 & 19 & 29.50 & 2.10 \\
\hline $29^{\circ}$ & 20 & 30.50 & 0.94 & 30 & 32.42 & 3.53 & 31 & 24.92 & -0.7 & 17 & 29.17 & 0.38 \\
\hline $30^{\circ}$ & 40 & 30.17 & -1.4 & 13 & 32.42 & -0.9 & 10 & 24.75 & 1.72 & 42 & 29.17 & -3.0 \\
\hline $31^{\circ}$ & 42 & 30.17 & -2.7 & 43 & 32.42 & 0.46 & 26 & 24.58 & -1.4 & 5 & 29.08 & 0.12 \\
\hline $32^{\circ}$ & 15 & 30.00 & -1.6 & 10 & 32.17 & 2.37 & 6 & 24.50 & -0.9 & 13 & 28.92 & -2.4 \\
\hline $33^{\circ}$ & 27 & 29.92 & -1.2 & 18 & 31.92 & -3.4 & 43 & 24.25 & -0.9 & 29 & 28.83 & -1.3 \\
\hline $34^{\circ}$ & 24 & 29.92 & -1.7 & 3 & 31.58 & -0.1 & 35 & 24.17 & -1.2 & 2 & 28.75 & -0.9 \\
\hline $35^{\circ}$ & 49 & 29.83 & 0.96 & 5 & 31.50 & 0.52 & 37 & 24.00 & 0.1 & 18 & 28.75 & -4.6 \\
\hline $36^{\circ}$ & 1 & 29.50 & 0.03 & 17 & 31.42 & 4.19 & 20 & 23.50 & -0.6 & 6 & 28.42 & -1.7 \\
\hline $37^{\circ}$ & 10 & 29.50 & 1.03 & 11 & 31.33 & -2.6 & 32 & 23.50 & -2.4 & 28 & 28.33 & -2.0 \\
\hline $38^{\circ}$ & 12 & 29.33 & -2.0 & 27 & 31.25 & -1.2 & 44 & 23.50 & -13 & 38 & 28.25 & 0.31 \\
\hline $39^{\circ}$ & 29 & 29.17 & -1.6 & 1 & 31.00 & 0.21 & 34 & 23.42 & -1.5 & 9 & 28.08 & -3.5 \\
\hline $40^{\circ}$ & 22 & 28.67 & -1.6 & 29 & 31.00 & -1.1 & 11 & 23.25 & -2.3 & 3 & 27.83 & -1.8 \\
\hline $41^{\circ}$ & 3 & 28.50 & -1.8 & 50 & 30.58 & 0.19 & 29 & 23.08 & -3.9 & 41 & 27.75 & -1.2 \\
\hline $42^{\circ}$ & 41 & 28.50 & -1.2 & 7 & 30.25 & -3.1 & 25 & 22.92 & -4.2 & 30 & 27.67 & 0.79 \\
\hline $43^{\circ}$ & 4 & 28.25 & -2.4 & 26 & 30.08 & -2.7 & 41 & 22.92 & -1.3 & 43 & 27.33 & -2.6 \\
\hline $44^{\circ}$ & 8 & 27.75 & -3.9 & 16 & 30.00 & -3.4 & 19 & 22.33 & -0.3 & 32 & 27.17 & -3.5 \\
\hline $45^{\circ}$ & 38 & 27.67 & -0.9 & 28 & 29.75 & -2.6 & 50 & 22.17 & -1.5 & 10 & 26.50 & -1.3 \\
\hline $46^{\circ}$ & 17 & 27.58 & -1.9 & 38 & 29.33 & -0.6 & 24 & 21.92 & -4.3 & 12 & 26.25 & -4.4 \\
\hline $47^{\circ}$ & 36 & 27.25 & -3.5 & 19 & 28.17 & -1.2 & 47 & 21.42 & -5.5 & 36 & 24.42 & 1.96 \\
\hline $48^{\circ}$ & 28 & 26.33 & -4.7 & 12 & 27.33 & -5.3 & 3 & 20.92 & -4.0 & 33 & 24.08 & -0.2 \\
\hline $49^{\circ}$ & 6 & 25.83 & -5.0 & 22 & 26.50 & -5.1 & 38 & 20.83 & -2.3 & 46 & 22.25 & -6.4 \\
\hline \multirow[t]{2}{*}{$50^{\circ}$} & 37 & 25.25 & -4.1 & 32 & 25.25 & -7.4 & 17 & 17.25 & 0.72 & 21 & 18.92 & -12 \\
\hline & & $\mathrm{D}=7.51$ & & & $D=19.92$ & & & $D=16.08$ & & & $D=25.2$ & \\
\hline
\end{tabular}


among the 50 produced with this tester, in descending order of yield, were the families 4, 9, 16, 23 and 33. Of the nine topcross hybrids from the IAC 125 that showed better average of GY, three were formed with families $(9,20$ and 33) which obtained high indices of GCA. In the tester UENF-14, three topcross hybrids with high average GY stood out, combined with families of high GCA effect, namely, families 2, 4 and 20 (Table 5). Thus, it can be said that the tester BRS Angela discriminated more consistently $\mathrm{S}_{3}$ families, according to their genetic merit for GY.

Among the nine hybrids showing better values of $\mathrm{PE}$, obtained with the tester BRS Angela, only one (sixth most expansive) was formed with a family of high index of GCA; the best family for such effect was the family 18 . For the tester IAC 125, three hybrids (fourth, seventh and ninth) of greater PE were formed by families that presented high GCA indices, the families 23, 25 and 9. The tester $\mathrm{P}_{2}$, as well as the tester BRS Angela, classified only one hybrid with high PE (sixth best hybrid) with the family 18 , which had the best GCA index for the trait. Among the hybrids from the UENF-14 tester with the highest values of PE, three were formed by families with significantly high GCA effects, the families 11, 25 and 47 (Table 5). The testers IAC 125 and UENF-14 stood out for having three topcross hybrids, classified with high $\mathrm{PE}$ indices within each group (tester), obtained from crossing with $\mathrm{S}_{3}$ families that held high indices of GCA. However, the hybrids from the tester IAC 125 provided a higher general average for the trait $\mathrm{PE}$ in relation to hybrids produced by crossings with the tester UENF-14. Therefore, the best tester for PE is the IAC 125 .

Table 5. Classification order of $\mathrm{S}_{3}$ families with respect to general combining ability and nine topcross hybrids with the highest indices with testers, for grain yield and popping expansion

\begin{tabular}{|c|c|c|c|c|c|c|c|c|c|c|c|c|c|}
\hline \multicolumn{7}{|c|}{ Grain yield } & \multicolumn{7}{|c|}{ Popping expansion } \\
\hline $\mathrm{S}_{3}$ & RG & $\mathbf{T 1}$ & T2 & T3 & T4 & CGC & $\mathbf{S}_{3}$ & CE & $\mathbf{T 1}$ & T2 & T3 & T4 & $\operatorname{cGC}$ \\
\hline 2 & 1106 & & & $7^{\circ}$ & $1^{\circ}$ & $7^{\circ}$ & 1 & 16.6 & & & $2^{\circ}$ & $5^{\circ}$ & \\
\hline 4 & 1087 & $1^{\circ}$ & & & $3^{\circ}$ & $3^{\circ}$ & 2 & 21.5 & & $2^{\circ}$ & $1^{\circ}$ & & \\
\hline 5 & 1972 & & $5^{\circ}$ & & & & 4 & 23.5 & & & & $3^{\circ}$ & \\
\hline 9 & 2585 & $2^{\circ}$ & $1^{\circ}$ & & & $1^{\circ}$ & 7 & 26.1 & $3^{\circ}$ & & $3^{\circ}$ & & \\
\hline 11 & 1640 & & $3^{\circ}$ & & & & 8 & 27.1 & & & $7^{\circ}$ & & \\
\hline 13 & 1202 & $3^{\circ}$ & & & & & 9 & 29.3 & & $7^{\circ}$ & & & $7^{\circ}$ \\
\hline 14 & 1397 & & $6^{\circ}$ & & & & 11 & 32.2 & & & & $6^{\circ}$ & $4^{\circ}$ \\
\hline 15 & 670 & $7^{\circ}$ & & & & & 13 & 29.3 & $8^{\circ}$ & & & & \\
\hline 16 & 1824 & $5^{\circ}$ & & & $7^{\circ}$ & $5^{\circ}$ & 14 & 23.2 & & & & $9^{\circ}$ & \\
\hline 18 & 817 & & & $6^{\circ}$ & & & 16 & 28.5 & $9^{\circ}$ & & & & \\
\hline 19 & 907 & & & & $2^{\circ}$ & & 18 & 35.2 & $6^{\circ}$ & & $6^{\circ}$ & & $1^{\circ}$ \\
\hline 20 & 1516 & & $2^{\circ}$ & & $4^{\circ}$ & $4^{\circ}$ & 19 & 28.6 & $5^{\circ}$ & & & & \\
\hline 22 & 1830 & $6^{\circ}$ & & & & & 20 & 19.9 & & & & $7^{\circ}$ & \\
\hline 23 & 1330 & $8^{\circ}$ & & & $9^{\circ}$ & $6^{\circ}$ & 21 & 32.7 & & $1^{\circ}$ & & & \\
\hline 24 & 1416 & & & $3^{\circ}$ & & & 23 & 30.4 & & $4^{\circ}$ & & & $2^{\circ}$ \\
\hline 30 & 576 & & & $8^{\circ}$ & & & 25 & 30.2 & & $9^{\circ}$ & & $2^{\circ}$ & $5^{\circ}$ \\
\hline 31 & 1814 & & $7^{\circ}$ & & & & 27 & 25.5 & & & & $8^{\circ}$ & \\
\hline 33 & 2051 & $9^{\circ}$ & $4^{\circ}$ & & & $2^{\circ}$ & 30 & 11.6 & & & $4^{\circ}$ & & \\
\hline 35 & 938 & & $8^{\circ}$ & & & & 31 & 22.9 & $4^{\circ}$ & & & & \\
\hline 36 & 1316 & & & $9^{\circ}$ & & & 39 & 15.5 & $2^{\circ}$ & $8^{\circ}$ & $5^{\circ}$ & & \\
\hline 37 & 1531 & & $9^{\circ}$ & & & & 40 & 25.2 & & & & $1^{\circ}$ & \\
\hline 41 & 1720 & & & $2^{\circ}$ & & & 42 & 34.2 & & & & & $3^{\circ}$ \\
\hline 43 & 1084 & & & $4^{\circ}$ & & & 44 & 25.2 & $1^{\circ}$ & $6^{\circ}$ & & & \\
\hline 44 & 1364 & & & & $8^{\circ}$ & & 45 & 16.2 & & $3^{\circ}$ & $9^{\circ}$ & & \\
\hline
\end{tabular}


Tabela 5. Continuation...

\begin{tabular}{|c|c|c|c|c|c|c|c|c|c|c|c|c|c|}
\hline \multicolumn{7}{|c|}{ Grain yield } & \multicolumn{7}{|c|}{ Popping expansion } \\
\hline $\mathrm{S}_{3}$ & RG & T1 & T2 & T3 & T4 & $\operatorname{cGC}$ & $\mathbf{S}_{3}$ & CE & T1 & T2 & T3 & T4 & CGC \\
\hline 46 & 1276 & & & & $6^{\circ}$ & & 46 & 20.0 & & & $8^{\circ}$ & & \\
\hline 47 & 1414 & & & & $5^{\circ}$ & & 47 & 30.2 & & & & $4^{\circ}$ & $6^{\circ}$ \\
\hline 49 & 709 & & & $1^{\circ}$ & & & 48 & 21.7 & $7^{\circ}$ & $5^{\circ}$ & & & \\
\hline 50 & 974 & $4^{\circ}$ & & $5^{\circ}$ & & & & & & & & & \\
\hline
\end{tabular}

\section{CONCLUSION}

The testers BRS Angela (for GY) and IAC 125 (for PE) were the most appropriate, when combined with $\mathrm{S}_{3}$ families derived from UENF-14 for the production of popcorn hybrids for the Northern and Northwestern Fluminense Regions.

\section{REFERENCES}

Amaral Júnior, A. T., Gonçalves, L. S. A., Freitas Júnior, S. P., Candido, L. S., Vitorazzi, C., Pena, G. F., Ribeiro, R. M., Silva, T. R. C., Pereira, M. G., Scapim, C. A., Viana, A. P. and Carvalho, G. F. (2013). UENF-14: a new popcorn cultivar. Crop Breeding and Applied Biotechnology, 13, 218. http://dx.doi.org/10.1590/S1984-70332013000300013.

Arnhold, E. and Milani, K. F. (2011). Rank-ordering coefficients of variation for popping expansion. Acta Scientiarum Agronomy, 33, 527-531. http://dx.doi.org/10.4025/ actasciagron.v33i3.11911.

Arnhold, E., Viana, J. M. S. and Silva, R. G. (2009). Associação de desempenho entre famílias $\mathrm{S}_{3}$ e seus híbridos topcross de milhopipoca. Revista Ciência Agronômica, 40, 396-399.

Barreto, R. R., Scapim, C. A., Amaral Júnior, A. T., Rodovalho, M. A., Vieira, R. A. and Schuelter, A. R. (2012). Avaliação da capacidade de combinação de famílias $S_{2}$ de milho-pipoca por meio de diferentes testadores. Semina: Ciências Agrárias, 33, 873-890. http://dx.doi. org/10.5433/1679-0359.2012v33n3p873.

Cruz, C. D. (2013). GENES - a software package for analysis in experimental statistics and quantitative genetics. Acta Scientiarum Agronomy, 35, 271-276. http://dx.doi.org/ 10.4025/actasciagron. v35i3.21251.

Davis, R. L. (1927). Report of plant breeder. Mayaguez: Puerto Rico Agricultural Experiment Station.

Fasoulas, A. C. (1983). Rating cultivars and trials in applied plant breeding. Euphytica, 32, 939-943. http://dx.doi.org/10.1007/ BF00042176.

Ferreira, E. A., Paterniani, M. E. A. G. Z., Duarte, A. P., Gallo, P. B., Sawazaki, E., Azevedo Filho, J.A. and Guimarães, P. S. (2009). Desempenho de híbridos topcrosses de linhagens $S_{3}$ de milho em três locais do Estado de São Paulo. Bragantia, 68, 319-327. http://dx.doi.org/10.1590/S0006-87052009000200005.

Fritsche Neto, R., Vieira, R. A., Scapim, C. A., Miranda, G. V. and Rezende L. M. (2012). Updating the ranking of the coefficients of variation from maize experiments. Acta Scientiarum Agronomy, 34, 99-101. http:// 10.4025/actasciagron.v34i1.13115.

Geraldi, I. O. and Miranda Filho, J. B. (1988). Adapted models for the analysis of combining ability of varieties in partial diallel crosses. Brazilian Journal of Genetics, 2, 419-430.

Granate, M. J., Cruz, C. D. and Pacheco, C. A. P. (2002). Predição de ganho genético com diferentes índices de seleção no milhopipoca cms-43. Pesquisa Agropecuária Brasileira, 37, 101-108. http://dx.doi.org/10.1590/S0100-204X2002000700014.

Griffing, A. R. (1956). Concept of general and specific combining ability in relation to diallel crossing system. Australian Journal of Biological Science, 9, 463-493.

Hallauer, A. R, Carena, M. J. and Miranda Filho, J. B. (2010). Quantitative genetics in maize breeding. lowa: Springer.

Jenkins, M. T. and Brunson, A. M. (1932). Methods of testing inbred lines of maize in crossbred combinations. Journal of the American Society of Agronomy, 24, 523-530.

Miranda Filho, J. B. and Gorgulho, E. P. (2001). Cruzamentos com testadores e dialelos. In L. L. Nas, A. C. C. Valois, I. S. Melo, and M. C. Valadares, Inglis recursos genéticos e melhoramento: plantas (p. 649-672). Rondonópolis: Fundação-MT.

Pereira, M. G. and Amaral Júnior, A. T. (2001). Estimation of genetic components in popcorn based on the nested design. 
Crop Breeding and Applied Biotechnology, 1, 3-10. http://dx.doi. org/10.13082/1984-7033.v01n01a01.

Pinto, R. J. B., Scapim, C. A., Ferreira Neto, A., Pacheco, C. A. P., Royer, M., Pedroni, M. V., Salvadori, R. K. and Silva, R. M. (2004). Analysis of testers of broad and narrow genetic base for topcrosses in popcorn breeding. Crop Breeding and Applied Biotechnology, 4, 152-162.

Rodovalho, M., Mora, F., Arriagada, O., Maldonado, C., Arnhold, E. and Scapim, C. A. (2014). Genetic evaluation of popcorn families using a Bayesian approach via the Independence chain algorithm. Crop Breeding and Applied Biotechnology, 14, 261-265. http://dx.doi.org/10.1590/1984-70332014v14n4n41.

Rodovalho, M., Scapim, C. A., Barth Pinto, R. J., Barreto,R. R., Ferreira, F. R. A. and Clóvis, L. R. (2012). Comparação de testadores em famílias $\mathrm{S}_{2}$ obtidas do híbrido simples de milho-pipoca IAC-112. Bioscience Journal, 28, 145-154.

Scapim, C. A., Carvalho, C. G. P. and Cruz, C. D. (1995). Uma proposta de classificação dos coeficientes de variação para a cultura do milho. Pesquisa Agropecuária Brasileira, 30, 683-686.

Scapim, C. A., Royer, M. R., Barth Pinto, R. J., Amaral Júnior, A. T., Pacheco, C. A. P. and Moterle, L. M. (2008). Comparison of testers in the evaluation of combining ability of $S_{2}$ families in popcorn. Revista Brasileira de Milho e Sorgo, 7, 83-91.

Seifert, A. L., Carpentieri-Pípolo, V., Ferreira, J. M. and Gerage, A. C. (2006). Análise combinatória de populações de milho-pipoca em topcrosses. Pesquisa Agropecuária Brasileira, 41, 771-778. http://dx.doi.org/10.1590/S0100-204X2006000500008.

Souza Neto, I. L., Barth Pinto, R. J., Scapim, C. A., Jobim, C. C., Figueiredo, A. S. T. and Bignotto, L. S. (2015). Diallel analysis and inbreeding depression of hybrid forage corn for agronomic traits and chemical quality. Bragantia, 74, 42-49. http://dx.doi.org/10.1590/1678-4499.0315.

Vencovsky, R. and Barriga, P. (1992). Genética biométrica no melhoramento. Ribeirão Preto: SBG.

Viana, J. M. S., Condé, A. B. T., Almeida, R. V., Scapim, C. A. and Valentini, L. (2007). Relative importance of per se and topcross performance in the selection of popcorn $\mathrm{S}_{3}$ families. Crop Breeding and Applied Biotechnology, 7, 74-81.

Yongbin, D., Zhongwei, Z., Qingling, S., Qilei, W., Qiang, Z. and Yuling, L. (2012). Quantitative trait loci mapping and meta-analysis across three generations for popping characteristics in popcorn. Journal of Cereal Science, 56, 581-586. http://dx.doi.org/10.1016/ j.jcs.2012.08.006. 